\title{
Research Article “Coffee Ring" Effect of Ag Colloidal Nanoparticles Dried on Glass: Impact to Surface-Enhanced Raman Scattering (SERS)
}

\author{
Petra Šimáková, Eva Kočišová, and Marek Procházka $\mathbb{D}$ \\ Charles University, Faculty of Mathematics and Physics, Institute of Physics, Ke Karlovu 5, CZ-121 16 Prague 2, Czech Republic \\ Correspondence should be addressed to Marek Procházka; prochaz@karlov.mff.cuni.cz
}

Received 4 June 2021; Accepted 5 August 2021; Published 18 August 2021

Academic Editor: Jianbo Yin

Copyright ( 2021 Petra Šimáková et al. This is an open access article distributed under the Creative Commons Attribution License, which permits unrestricted use, distribution, and reproduction in any medium, provided the original work is properly cited.

\begin{abstract}
It is well known that spontaneous drying of some fluid droplets on certain solid surfaces forms a "coffee ring" pattern. In this paper, we studied "coffee ring" formation for two kinds of Ag colloidal nanoparticles (borohydride-reduced (b.-r.) and hydroxylamine-reduced (h.-r.)) and its impact on surface-enhanced Raman scattering (SERS). Optical and scanning electron microscopies were used to observe the morphology of the dried rings as well. We used 5,10,15,20-tetrakis(1-methyl-4-pyridyl)porphyrin (TMPyP) as a testing SERS molecular probe. The results showed that the structure of the edge rings of dried drops of Ag colloid/TMPyP systems was different for b.-r. and h.-r. nanoparticles. The inherent limitation of our approach is inhomogeneity in particle and "hot spots" distribution, SERS signal fluctuation, and consequently low spectral reproducibility. However, in the case of h.-r. nanoparticles, it formed a structure with highly enhancing sites ("hot spots") providing enormous SERS signal of TMPyP. Higher sensitivity and the possibility of spectral mapping over the dried pattern are advantages in comparison with the measurements from colloidal suspension. Although our approach is not reliable for quantitative analytical SERS applications, it can serve as a simple, cheap, and fast prescan method, which can be easily implemented for preliminary SERS analysis.
\end{abstract}

\section{Introduction}

It is well known that a spontaneous drying of a fluid droplet on a solid surface at ordinary room temperature causes a fluid movement radially outward to the contact line in order to maintain its position. In some cases, it can lead to the formation of a ring of deposited material in the outer part of the dried pattern, which is denoted as a "coffee ring" effect [1]. The created "coffee ring" is a result of an interplay of contact line pinning, solvent evaporation, and capillary flow [2]. The well-shaped compact rings are formed for a variety of substrates (mostly hydrophobic) [3-6] and dispersed materials [3-8]. In the case of micro- or nanoparticles, "coffee ring" was first observed for charged polystyrene microsphere drops [7] and later for metal colloidal nanoparticles deposited and dried on hydrophilic substrates such as a glass slide [8].

$\mathrm{Au}$ and $\mathrm{Ag}$ nanoparticles are frequently used as active substrates for surface-enhanced Raman scattering (SERS) [9]. SERS is a useful detection and analytical technique based on an enormous enhancement of Raman scattering (usually up to $10^{6}$ ) from molecules adsorbed on a roughened metal surface (so-called "SERS-active substrate") [9]. In the case of $\mathrm{Au}$ or $\mathrm{Ag}$ nanoparticles, high SERS enhancement is obtained when they form aggregates containing highly enhancing sites "hot spots." Drying by a "coffee-ring" effect promotes adsorption of the studied analytes to nanoparticle surface as well as aggregation and packing of nanoparticles in the ring at the edge of the droplet $[10,11]$. This results in high SERS enhancement but low spectral reproducibility. Such approach is simple and was successfully used for SERS detection of lipids [11], proteins [12], and uric acid [13].

Higher uniformity and spectral reproducibility of dried nanoparticles can be obtained by control of drying properties in order to avoid the "coffee-ring" effect. This was done in the case of nanoparticles dropped and dried on aluminium oxide surface [13] or wrinkle-confined surface [14]. On the other hand, the enhancement factor was low $\left(10^{4}\right)$, hence the same as for measurement directly from colloidal suspensions [13]. Close packing of colloidal nanoparticles can be achieved by self-assemblies of nanoparticles immobilized onto glass slide via organosilanes [15] or densely packed interfacial nanoparticle 2D metal-like liquid films (MeLLFs) formed in 


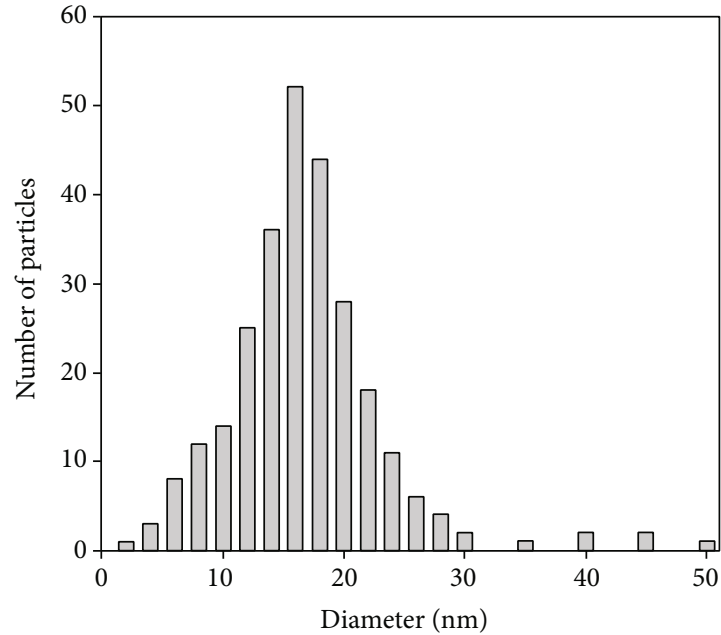

(a)



(b)

FIgUre 1: Particle size distribution of b.-r. (a) and h.-r. (b) Ag colloid determined from SEM images.

liquid/liquid interface [16]. Higher structural control of selfassemblies is possible by modern techniques [17] such as template-assisted self-assembly [18] including supercrystallike arrangement $[19,20]$. These approaches provide uniform and discrete assemblies; however, their preparation methods are inherently challenging and both time- and cost-consuming.

In this paper, we study the "coffee ring" formation in the case of two kinds of Ag nanoparticles (borohydride-reduced (b.-r.) and hydroxylamine-reduced (h.-r.)) deposited and dried on clean glass slides. We concentrate primarily on its impact on SERS activity, sensitivity, and reproducibility. Optical microscopy and SEM were used to observe the morphology of the dried ring. We used 5,10,15,20-tetrakis (1-methyl-4-pyridyl)porphyrin (TMPyP) as a testing SERS molecular probe. We will discuss the usefulness of the proposed approach for SERS. We will focus on its advantages and limitations in comparison to the SERS measurements directly from colloidal suspensions as well as from more controlled assemblies of colloidal nanoparticles.

\section{Materials and Methods}

Deionized water and pure chemicals purchased from SigmaAldrich were used. Ag colloid was prepared by reduction of $\mathrm{AgNO}_{3}$ with sodium borohydride (b.-r.) [21] and with hydroxylamine hydrochloride (h.-r.) [22]. The maximum of extinction spectrum is at $394 \mathrm{~nm}$ and $414 \mathrm{~nm}$ for freshly prepared b.-r. and h.-r. colloid, respectively. Ag colloid/TMPyP SERS-active systems were prepared by the addition of TMPyP to the Ag colloid to obtain the desired concentration of TMPyP $\left(1 \times 10^{-6} \mathrm{M}-1 \times 10^{-10} \mathrm{M}\right)$. Immediately after preparation, $\sim 2 \mu \mathrm{L}$ of the mixture was dropped by pipette on a cleaned (by piranha solution and ethanol) microscope glass slide $(75 \times 26 \mathrm{~mm})$. The droplet was left to dry at room temperature for about half an hour.

SERS spectra were recorded with an integrated confocal Raman microscopic system LabRam HR800 (Horiba Jobin Yvon) with a nitrogen-cooled CCD detector using the
$514.5 \mathrm{~nm}$ excitation line of an $\mathrm{Ar}^{+}$laser $(\sim 0.2 \mathrm{~mW}$ at the sample) and acquisition time $1 \mathrm{~s}$. Bright-field images of the dried drops were taken by the built-in digital camera using an optical microscope (Olympus BX) objectives 5x and 50x.

Extinction spectra were measured by UV-Vis spectrometer Perkin Elmer Lambda 12. The scanning electron microscopy (SEM) images were obtained by scanning electron microscope JEOL JSM-7500F. The maximum of particle size distribution determined by SEM is at $16.8 \mathrm{~nm}$ and $38 \mathrm{~nm}$ for b.-r. and h.-r. colloid, respectively (Figure 1).

\section{Results}

3.1. "Coffee Ring" Formation and Its Morphology. When the drops of Ag colloid/TMPyP system were deposited carefully on clean glass slides and dried, both the drops with b.-r. and h.-r. colloidal nanoparticles had a tendency to form a ring in the edge part of the dried droplet in which most nanoparticles and their aggregates were accumulated. The optical microscopy (bright-field) images for b.-r. and h.-r. Ag colloid/TMPyP $\left(1 \times 10^{-7} \mathrm{M}\right)$ systems are shown in Figure 2 . The whole dried pattern has a round shape with a diameter about $1000-1400 \mu \mathrm{m}$ and a well-distinguished and compact edge ring (see Figure 2(a) for h.-r. colloid). The details of the edge of the rings are compared in Figures 2(b) and 2(c) for both kinds of nanoparticles. The formation of the ring was observed for all measured TMPyP concentrations although the rings had slightly different widths and were not always compact. The nanoparticle aggregates several microns in sizes can be seen in the region toward the center of the dried drop, but there were completely absent in its central part (Figure 2(a)).

More detailed information about the morphology of the edge rings of dried drops was obtained by electron microscopy. SEM images of the edge rings of b.-r. and h.-r. Ag colloid/TMPyP $\left(1 \times 10^{-7} \mathrm{M}\right)$ systems are shown in Figure 3 . They indicate that the aggregates in the "coffee ring" were quite diverse. In the case of b.-r. colloid, the ring was formed 


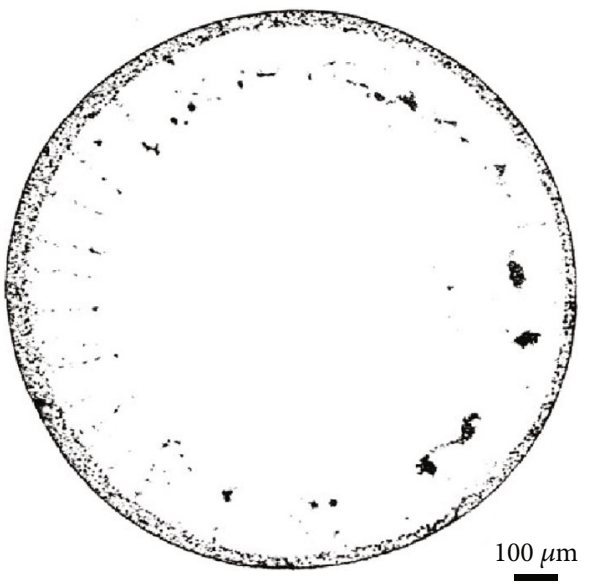

(a)

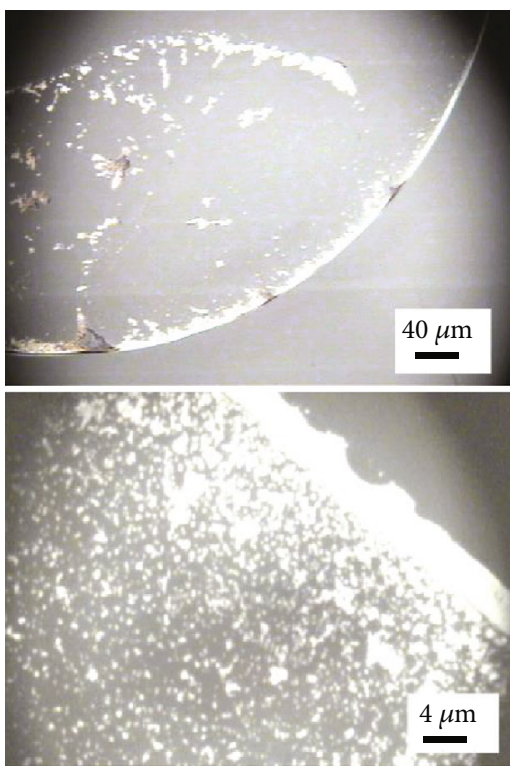

(b)

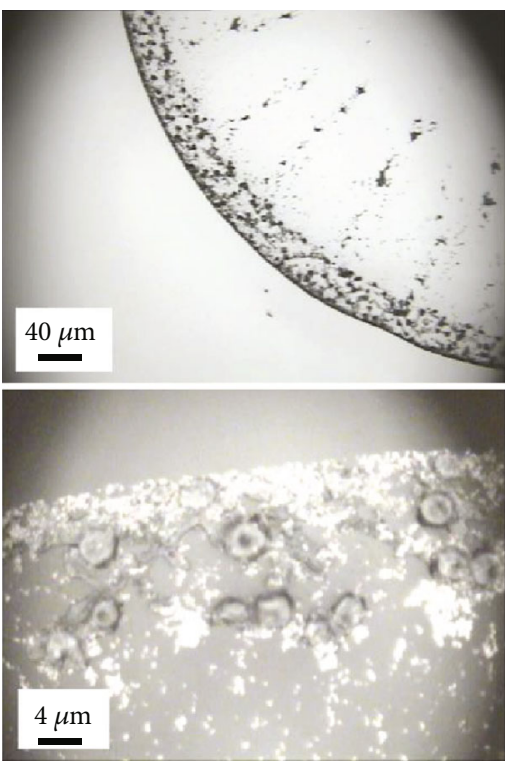

(c)

FIGURE 2: Optical microscopy (bright-field) images of Ag colloid/TMPyP $\left(1 \times 10^{-7} \mathrm{M}\right)$ systems dried on glass slide: (a) the whole ring for h.-r. colloid; (b) the edge ring for b.-r. colloid; (c) the edge ring for h.-r. colloid.

by very closely packed nanoparticles at the edge (about $10 \mu \mathrm{m}$ width) and then small aggregates or isolated particles decreasing toward the center of the drop. On the other hand, in the case of h.-r. colloid, the nanoparticles were not well packed; the ring was about $10 \mu \mathrm{m}$ wide and contained large aggregates of nanoparticles or alternating empty spaces. Moreover, many large crystals of salt (several $\mu \mathrm{m}$ in diameter) coming from the preparation procedure were presented in the case of h.-r. nanoparticles (Figures 2(c) and 3(b)).

\subsection{SERS Spectra of TMPYP and Enhancement Factor.} Figure 4 shows extinction spectra of Ag colloid/TMPyP $\left(1 \times 10^{-7} \mathrm{M}\right)$ systems dried on a glass slide for b.-r. (dotted line) and h.-r. (solid line) colloid. The maxima at 394 and $414 \mathrm{~nm}$ correspond to surface plasmon extinction bands of b.-r. and h.-r. colloid, respectively. $514.5 \mathrm{~nm}$ excitation wavelength was chosen for SERS measurements because of the localized surface plasmon resonance effect at this region.
For SERS spectral testing, first, we measured SERS spectra of TMPyP (see its chemical structure, Figure 5(a)) from ring patterns. Figure 5(b) shows typical SERS spectra obtained from a dried h.-r. colloid/TMPyP system where TMPyP concentration varies from $1 \times 10^{-6} \mathrm{M}$ to $1 \times 10^{-10}$ $M$. Since the SERS spectra were strongly influenced by fluctuations in signal intensity and signal distribution, we tried to find a suitable position for spectral acquisition providing the highest spectral intensity. This is the reason why the spectra do not show a clear trend of intensity decrease with TMPyP concentration. On the other hand, lower TMPyP concentration in the SERS-active system can be seen through the strong band at $240 \mathrm{~cm}^{-1}$ corresponding to $\mathrm{AgCl}$. $\mathrm{Cl}$ anions come from a hydroxylamine hydrochloride reduction agent. Raman band of $\mathrm{AgCl}$ significantly increased in the spectra with a decrease of TMPyP concentration because less $\mathrm{Cl}$ anions were replaced by TMPyP. Spectral features of TMPyP porphyrin were observed even for $1 \times 10^{-10} \mathrm{M}$ concentration 


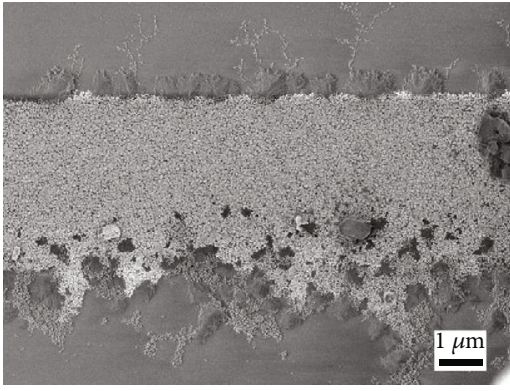

(a)

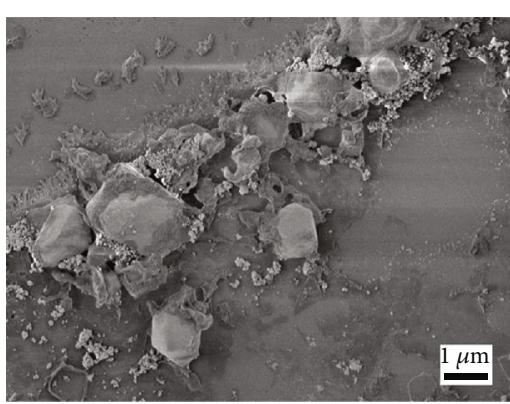

(b)

Figure 3: SEM images for Ag colloid/TMPyP $\left(1 \times 10^{-7} \mathrm{M}\right)$ systems dried on a glass slide for (a) b.-r. colloid and (b) h.-r. colloid.

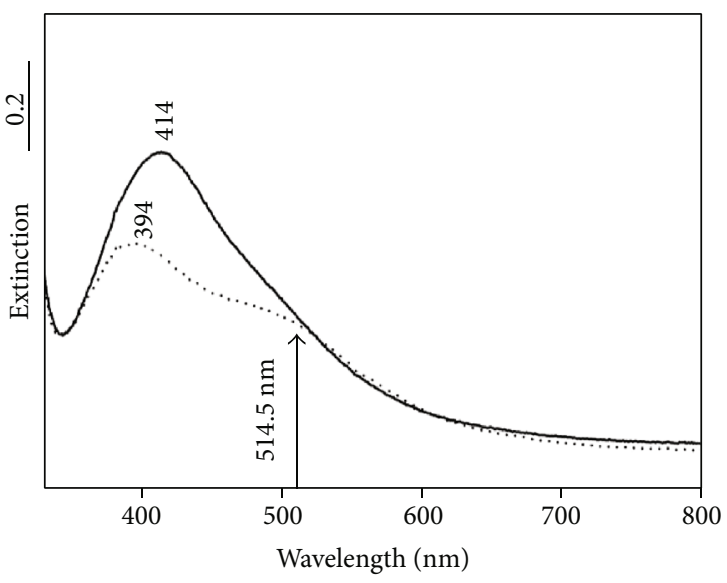

Figure 4: Extinction spectra of Ag colloid/TMPyP $\left(1 \times 10^{-7} \mathrm{M}\right)$ systems dried on a glass slide for b.-r. (dotted line) and h.-r. (solid line) colloid. The excitation wavelength used for SERS measurement is marked by an arrow.

although some new bands originating from carbon species contamination [23] occurred as well. The SERS spectra of TMPyP were also obtained for b.-r. Ag colloid/TMPyP systems dried on a glass slide (not shown here), but the lowest detectable concentration was $1 \times 10^{-7} \mathrm{M}$.

It is known that the free-base porphyrins can be metalated by the incorporation of silver ions into their macrocycle [21]. Thus, the SERS spectra of TMPyP were a mixture of two spectral forms: free-base (spectral markers at ca. 330, $967+1000,1335 \mathrm{~cm}^{-1}$ ) and metalated form (spectral markers at ca. 395, 1010,1340 $\mathrm{cm}^{-1}$ ) [24]. The vibrational assignment can be found at [25]. The degree of metalation strongly depends on TMPyP concentration and time between preparation of SERS-active system and SERS measurement $[24,26]$. Therefore, the ratio of the free-base and the metalated TMPyP forms varied from spectrum to spectrum in Figure 5(b).

We tried to estimate an analytical enhancement factor (AEF) [9] of our dried Ag colloidal nanoparticles. It is expressed by the intensity ratio of SERS and Raman spectra multiplied by the corresponding concentration ratio. For calculation, we used SERS spectra of $1 \times 10^{-7} \mathrm{M}$ TMPyP concentration which is in submonolayer adsorption limit [24] and Raman spectrum of $1 \times 10^{-3} \mathrm{M}$ TMPyP dried on glass slide without Ag nanoparticles measured under the same experimental conditions. The AEF for TMPyP was about $10^{5}$ for both b.-r. and h.-a. nanoparticles. In the case of h-a. nanoparticles, when we used the lowest detectable SERS spectrum $\left(1 \times 10^{-10} \mathrm{M}\right.$ TMPyP concentration $)$ for calculation, AEF reached $10^{8}$ orders of magnitude. Because of the strong fluctuation of SERS intensity for this concentration, this AEF is a rough estimation.

3.3. Reproducibility of SERS Measurements. To check uniformity and reproducibility of SERS measurements, we carried out SERS mapping experiments where the spectra were measured from a set of selected points as seen in Figures 6(a) and 6(b) (left part). The increment between mapping points was 5 and $10 \mu \mathrm{m}$ for b.-r. and h.-r. nanoparticles, respectively. Figure 6 shows typical spectral maps for both b.-r. and h.-r. Ag colloid/TMPyP $\left(1 \times 10^{-7} \mathrm{M}\right)$ systems. The obtained sets of spectra were treated by factor analysis using the "singular value decomposition" algorithm [24]. The intensity in each mapping point was determined as the coefficient of the first subspectrum of the factor analysis. The colour scale represents SERS intensity normalized for each set of spectra. The intensities were normalized for each set of spectra but not for both spectral sets together. The results indicate that the ring was not a homogeneous pattern, and the intensity for both systems strongly varied from point to point. Certain points (probably "hot spots") showed 5-10 times higher intensity than the vast majority of other points. As observed, different morphology of aggregates formed in the edge ring of both kinds of nanoparticles had no significant influence on the variation of spectral intensities.

\section{Discussion}

Our results demonstrate that dried drops of b.-r. and h.-a. Ag colloid/TMPyP systems formed compact "coffee rings" after drying on glass slides. The optical microscopy (bright-field) and SEM images proved that the nanoparticle aggregates were accumulated in the edge rings. It is necessary to mention that $1 \times 10^{-7} \mathrm{M}$ TMPyP concentration was under submonolayer adsorption limit [24], and consequently, SERS-active system was only slightly aggregated. Therefore, strong aggregation was not produced by adding TMPyP but by the drying process itself. Since drying was spontaneous and uncontrolled, the aggregates were quite diverse. Our results show that drying led to different morphology of the 


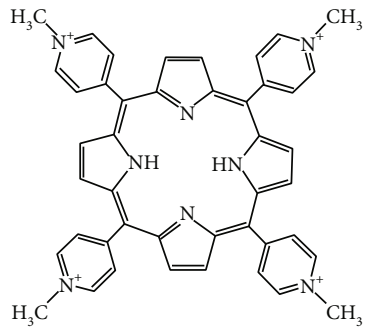

(a)

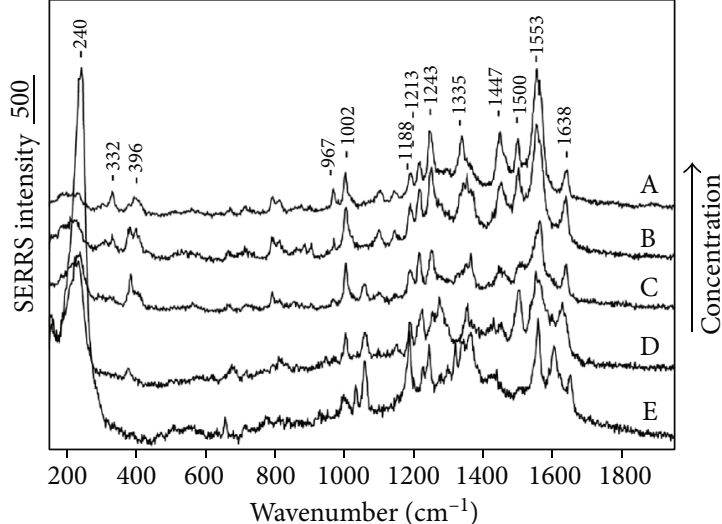

(b)

Figure 5: (a) Chemical structure of TMPyP and (b) typical SERS spectra obtained from the dried h.-r. Ag colloid/TMPyP system. Concentrations of TMPyP in the system: (A) $1 \times 10^{-6}$; (B) $1 \times 10^{-7}$; (C) $1 \times 10^{-8}$; (D) $1 \times 10^{-9}$; (E) $1 \times 10^{-10} \mathrm{M}$.
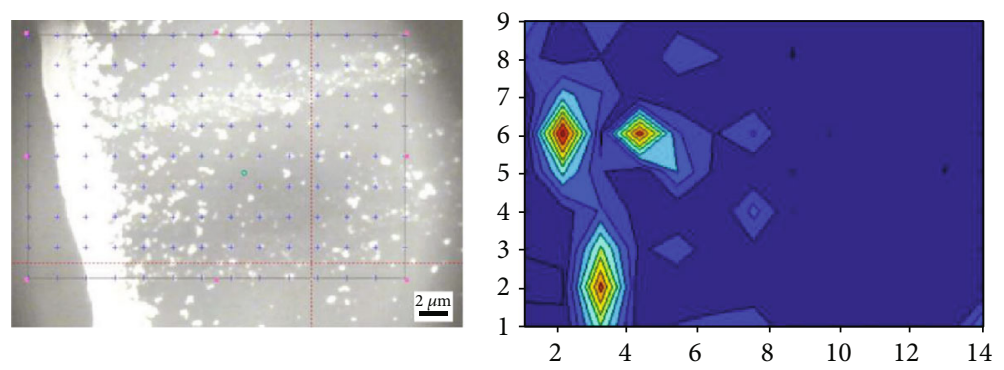

(a)
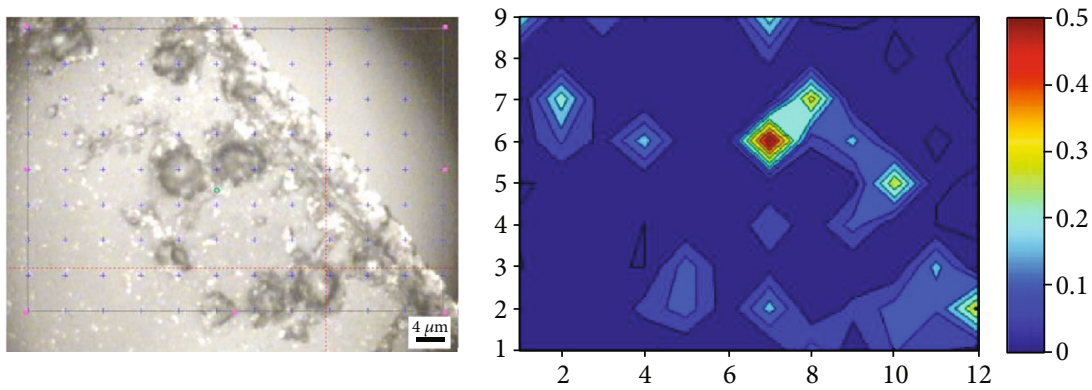

(b)

FIGURE 6: Optical microscopy (bright-field) images and corresponding spectral maps for b.-r. and h.-r. Ag colloid/TMPyP $\left(1 \times 10^{-7} \mathrm{M}\right)$ system (a) and (b), respectively. Numbers on the $x$ and $y$ axes mean spectral points; colour scale represents normalized SERS intensity.

edge ring in the case of b.-r. and h.-r. nanoparticles, because of different particle sizes and different concentrations and kinds of residual ions at the nanoparticle surface. The b.-r. nanoparticles were very closely packed in the ring while in the case of h.-r. colloid, the ring contained large aggregates of nanoparticles.

Concerning SERS spectral testing, SERS sensitivity of dried b.-r. and h.-r. nanoparticles accumulated in the edge rings was different, due to their different morphology and aggregation state. The lowest detectable concentration of TMPyP was $1 \times 10^{-7} \mathrm{M}$ and $1 \times 10^{-10} \mathrm{M}$ for b.-r. and h.-a. nanoparticles, respectively. A similar spectral detection limit $\left(5 \times 10^{-8} \mathrm{M}\right)$ was previously reported for TMPyP adsorbed on b.-r. nanoparticles attached to a glass slide by organosilane [15]. As for colloidal suspension, the spectral detection limits of TMPyP under the same excitation wavelength were about $1 \times 10^{-7} \mathrm{M}$ and $1 \times 10^{-8} \mathrm{M}$, for b.-r. and h.-a. colloid, respectively. It is known that an average AEF for nonaggregated or slightly aggregated Ag colloidal nanoparticles in suspension is about $10^{4}$ and for Ag nanoparticles and nanostructures in a dry state about $10^{4}$ to $10^{6}[13,27]$. For comparison, our AEF obtained for h.-a. for colloid/TMPyP system dried to "coffee ring" pattern was estimated to $10^{8}$ in order of magnitude. Therefore, it is evident that dried h.-r. nanoparticles accumulated in the edge ring formed a morphology with many aggregates containing "hot spots" 
providing high SERS signal of TMPyP. This can be considered an advantage of such SERS-active systems in comparison with the colloidal suspensions themselves or the $2 \mathrm{D}$ nanoparticle assemblies.

The SERS spectra measured from the dried "coffee ring" pattern did not show a clear trend of intensity decrease with TMPyP concentration. In addition, the spectral mapping revealed strong fluctuations of signal intensity and the signal distribution from spot to spot. It is evident that besides the rationally designed $2 \mathrm{D}$ nanoparticle assemblies, the aggregates formed by spontaneous drying were rather undefined with an uncontrolled particle density, inhomogeneity in particle, and "hot spot" distribution, and consequently, they provided low spectral reproducibility. It is the inherent limitation of this approach that is, hence, not reliable for quantitative analytical SERS applications. On the other hand, higher sensitivity and the possibility of finding the highest signal by spectral mapping over the dried pattern are advantages over the measurements from colloidal suspension. Therefore, we propose that our approach can serve as a simple, cheap, and fast prescan method, which can be easily implemented for preliminary SERS analysis.

\section{Conclusions}

In this paper, we studied "coffee ring" effect of two kinds of Ag nanoparticles (b.-r. or h.-r.) and its impact on SERS activity using TMPyP porphyrin molecular probe. The results proved that dried drops of Ag colloid/TMPyP systems on glass slides formed compact rings containing nanoparticle aggregates. The morphology of the aggregates was different for b.-r. and h.-r. colloids. It led to higher SERS sensitivity for h.-r. nanoparticles $\left(1 \times 10^{-10} \mathrm{M}\right.$ TMPyP concentration was detected) but did not affect spectral intensity variations observed in both cases. The inherent limitation of our approach is inhomogeneity in particle and "hot spots" distribution, SERS signal fluctuation, and consequently low spectral reproducibility. On the other hand, higher sensitivity and the possibility of spectral mapping over the dried pattern are advantages in comparison with the measurements from colloidal suspension. Although our approach is not reliable for quantitative analytical SERS applications, it can serve as a simple, cheap, and fast prescan method, which can be easily implemented for preliminary SERS analysis.

\section{Data Availability}

The data used to support the findings of this study are available from the corresponding author upon request.

\section{Conflicts of Interest}

The authors declare that there is no conflict of interests regarding the publication of this paper.

\section{Acknowledgments}

SEM images were carried out at the Department of Physical Electronics, Faculty of Nuclear Sciences and Physical Engi- neering, Czech Technical University in Prague (Dr. Jan Proška). The authors acknowledge the financial support from the Czech Science Foundation (18-10897S) and the Charles University Research Centre program UNCE/SCI/010.

\section{References}

[1] R. D. Deegan, O. Bakajin, T. F. Dupont, G. Huber, S. R. Nagel, and T. A. Witten, "Capillary flow as the cause of ring stains from dried liquid drops," Nature, vol. 389, no. 6653, pp. 827829, 1997.

[2] R. D. Deegan, "Pattern formation in drying drops," Physical Review E, vol. 61, no. 1, pp. 475-485, 2000.

[3] D. Zhang, Y. Xie, M. F. Mrozek, C. Ortiz, V. J. Davisson, and D. Ben-Amotz, "Raman detection of proteomic analytes," Analytical Chemistry, vol. 75, no. 21, pp. 5703-5709, 2003.

[4] E. Kočišová, M. Petr, H. Šípová, O. Kylián, and M. Procházka, "Drop coating deposition of a liposome suspension on surfaces with different wettabilities: "coffee ring" formation and suspension preconcentration," Physical Chemistry Chemical Physics, vol. 19, no. 1, pp. 388-393, 2017.

[5] E. Kočišová, M. Procházka, and H. Šípová, “Thiol-modified gold-coated glass as an efficient hydrophobic substrate for drop coating deposition Raman (DCDR) technique," Journal of Raman Spectroscopy, vol. 47, no. 11, pp. 1394-1396, 2016.

[6] E. Kočišová and M. Procházka, "Drop-coating deposition Raman spectroscopy of liposomes," Journal of Raman Spectroscopy, vol. 42, pp. 1606-1610, 2011.

[7] J.-Y. Jung, Y. W. Kim, and J. Y. Yoo, "Behavior of particles in an evaporating didisperse colloid droplet on a hydrophilic surface," Analytical Chemistry, vol. 81, no. 19, pp. 8256-8259, 2009.

[8] A. F. Routh, "Drying of thin colloidal films," Reports on Progress in Physics, vol. 76, no. 4, article 046603, 2013.

[9] M. Procházka, "Surface-Enhanced Raman Spectroscopy: Bioanalytical," in Biomolecular and Medical Applications, Springer International Publishing, Switzerland, 2016.

[10] P. Šimáková, M. Procházka, and E. Kočišová, "SERS microspectroscopy of biomolecules on dried Ag colloidal drops," Spectroscopy: An International Journal, vol. 27, pp. 449-453, 2012.

[11] P. Šimáková, E. Kočišová, and M. Procházka, "Sensitive Raman spectroscopy of lipids based on drop deposition using DCDR and SERS," Journal of Raman Spectroscopy, vol. 44, no. 11, pp. 1479-1482, 2013.

[12] S. Keskin and M. Culha, "Label-free detection of proteins from dried-suspended droplets using surface enhanced Raman scattering," Analyst, vol. 137, no. 11, pp. 2651-2657, 2012.

[13] M. Pucetaite, M. Velicka, J. Pilipavicius, A. Beganskiene, J. Ceponkus, and V. Sablinskas, "Uric acid detection by means of SERS spectroscopy on dried Ag colloidal drops," Journal of Raman Spectroscopy, vol. 47, no. 6, pp. 681-686, 2016.

[14] N. Pazos-Pérez, W. Ni, A. Schweikart, R. A. Alvarez-Puebla, A. Fery, and L. M. Liz-Marzán, "Highly uniform SERS substrates formed by wrinkle-confined drying of gold colloids," Chemical Science, vol. 1, no. 2, pp. 174-178, 2010.

[15] N. Hajduková, M. Procházka, P. Molnár, and J. Štěpánek, "SERRS of free-base porphyrins on immobilized metal gold and silver nanoparticles," Vibrational Spectroscopy, vol. 48, no. 1, pp. 142-147, 2008. 
[16] M. P. Konrad, A. P. Doherty, and S. E. J. Bell, "Stable and uniform SERS signals from self-assembled two-dimensional interfacial arrays of optically coupled Ag nanoparticles," Analytical Chemistry, vol. 85, no. 14, pp. 6783-6789, 2013.

[17] C. Kuttner, "Plasmonic in Sensing: From Colorimetry to SERS Analytics," in Plasmonic, T. Gric, Ed., IntechOpen, 2018.

[18] M. Rycenga, P. H. C. Camargo, and Y. Xia, “Template-assisted self-assembly: a versatile approach to complex micro- and nanostructures," Soft Matter, vol. 5, no. 6, pp. 1129-1136, 2009.

[19] C. Matricardi, C. Hanske, J. L. Garcia-Pomar, J. Langer, A. Mihi, and L. M. Liz-Marzán, "Gold nanoparticle plasmonic superlattices as surface-enhanced Raman spectroscopy substrates," ACS Nano, vol. 12, no. 8, pp. 8531-8539, 2018.

[20] M. Tebbe, S. Lentz, L. Guerrini, A. Fery, R. A. Alvarez-Puebla, and N. Pazos-Perez, "Fabrication and optical enhancing properties of discrete supercrystals," Nanoscale, vol. 8, no. 25, pp. 12702-12709, 2016.

[21] B. Vlckova, P. Matejka, J. Simonova, K. Cermakova, P. Pancoska, and V. Baumruk, "Surface-enhanced resonance Raman spectra of free base 5,10,15,20-tetrakis(4-carboxyphenyl)porphyrin and its silver complex in systems with silver colloid: direct adsorption in comparison to adsorption via molecular spacer," The Journal of Physical Chemistry, vol. 97, no. 38, pp. 9719-9729, 1993.

[22] N. Leopold and B. Lendl, "A new method for fast preparation of highly surface-enhanced Raman scattering (SERS) active silver colloids at room temperature by reduction of silver nitrate with hydroxylamine hydrochloride," The Journal of Physical Chemistry. B, vol. 107, no. 24, pp. 5723-5727, 2003.

[23] A. Kudelski, "Some aspects of SERS temporal fluctuations: analysis of the most intense spectra of hydrogenated amorphous carbon deposited on silver," Journal of Raman Spectroscopy, vol. 38, no. 11, pp. 1494-1499, 2007.

[24] M. Procházka, J. Štěpánek, P.-Y. Turpin, and J. Bok, “Drastically different porphyrin adsorption and metalation processes in chemically prepared and laser-ablated SERS-active silver colloidal substrates," The Journal of Physical Chemistry. B, vol. 106, no. 7, pp. 1543-1549, 2002.

[25] J. Hanzlíková, M. Procházka, J. Štěpánek, J. Bok, V. Baumruk, and P. Anzenbacher, "Metalation of 5,10,15,20-tetrakis(1methyl-4-pyridyl)porphyrin in silver colloids studied via time dependence of surface-enhanced resonance Raman spectra," Journal of Raman Spectroscopy, vol. 29, no. 7, pp. 575-584, 1998.

[26] M. Procházka, J. Hanzliková, J. Štěpánek, and V. Baumruk, "Metalation of positively charged water soluble mesoporphyrins studied via time-resolved SERRS spectroscopy," Journal of Molecular Structure, vol. 410-411, pp. 77-79, 1997.

[27] R. Höller, I. J. Jahn, D. Cialla-May et al., "Biomacromolecularassembled nanoclusters: key aspects for robust colloidal SERS sensing," ACS Applied Materials \& Interfaces, vol. 12, no. 51, pp. 57302-57313, 2020. 